\title{
Subgraph with Set Similarity in Adatabase
}

\author{
Vinjamuri Kantha Rao1), Ronnie Caytiles²)
}

\begin{abstract}
In real-world graphs like social networks, linguistics net and biological networks, every vertex typically contains wealthy info, which might be shapely by a group of tokens or parts. during this paper, we have a tendency to study a subgraph matching with set similarity (SMS2) question over an outsized graph information, that retrieves subgraphs that ar structurally isomorphous to the question graph, and in the meantime satisfy the condition of vertex try matching with the (dynamic) weighted set similarity. To with efficiency method the SMS2 question, this paper styles a unique lattice-based index for knowledge graph, and light-weight signatures for each question vertices and knowledge vertices. supported the index and signatures, we have a tendency to propose associate economical two-phase pruning strategy as well as set similarity pruning and structure-based pruning, that exploits the distinctive options of each (dynamic) weighted set similarity and graph topology. we have a tendency to conjointly propose associate economical dominating-set-based subgraph matching formula radio-controlled by a dominating set choice formula to realize higher question performance. in depth experiments on each real and artificial datasets demonstrate that our technique outperforms progressive strategies by associate order of magnitude.
\end{abstract}

Keywords: Isomorphous, Satisfy, Performance, Progressive, Topology, Datasets.

\section{EXISTING SYSTEM:}

Exact subgraph matching question needs that each one the vertices's and edges ar matched precisely. The Ullman's subgraph isomorphy technique formula don't utilize any index structure, so they're typically pricey for giant graphs. Tree $\mathrm{Pi}$ indexes graph databases mistreatment frequent

subtrees as classification structures. cushion may be a structure distance primarily based subgraph matching formula in an exceedingly massive graph. Chao et AL. investigated the $S$ Path formula, that utilizes shortest methods round the vertex as basic index units. Cheng et $\mathrm{AL}$ planned a brand new ballroom dancing $\mathrm{R}$ - be part of formula to with efficiency realize matching graph patterns from an outsized graph. Zou et al. planned a distancebased[1] multi-way be part of formula for responsive pattern match queries over an outsized graph.

Received(January 31, 2017), Review Result(1st: February 28, 2017, 2nd: April 28 2017), Accepted(May 10, 2017)

${ }^{1}$ Department of Computer Science and Engineering KL University, Vaddeswaram, Guntur mail2kanthu09@kluniversity.in

${ }^{2}$ Dept. of Multimedia, Hannam University, 70, Hannam-ro, Daedeok-gu, Daejeon, Korea, rdcaytiles@gmail.com 
Shang et al. planned QuickSI formula for subgraph isomorphy optimized by selecting associate search order supported some options of graphs. SING may be a novel classification system for subgraph isomorphy in an exceedingly massive scale graph. GraphQL may be a search language for graph databases that supports graphs because the basic unit of data. Sun et al. utilised graph exploration and parallel computing to method subgraph matching question on a billion node graph. Recently, associate economical and sturdy subgraph isomorphy formula TurboISO was planned. RINQ ar graph alignment algorithms for biological networks, which might be accustomed solve isomorphy issues. However, a question graph is far smaller than the information graph in subgraph isomorphy issues, whereas the 2 graphs typically have similar size in graph alignment issues. to resolve subgraph isomorphy issues, graph alignment[2] algorithms introduce further value as they ought to 1st realize candidate subgraphs of comparable size from the massive knowledge graph. additionally, existing actual subgraph matching and graph alignment algorithms don't contemplate weighted set similarity on vertices, which can cause high postprocessing value of set similarity computation.

\section{PROPOSED SYSTEM:}

Due to the unskillfulness of existing strategies, we have a tendency to propose associate economical SMS2 question process approach during this paper. Our approach adopts a "filterand-refine" framework, that exploits distinctive options of each graph topology and dynamic weighted set similarity. within the filtering part, we have a tendency to build a lattice-based index over frequent patterns of component sets of vertices in knowledge graph G. Then, knowledge vertices ar encoded into signatures, and arranged into signature buckets. we have a tendency to style associate economical two-phase pruning strategy supported the lattice-based index and signature buckets to cut back the SMS2 search house. within the refinement part, we have a tendency to propose a dominating set 2(DS)-based subgraph matching formula to search out subgraph matches with set similarity (defined in Definition 1). A dominating set choice technique is planned to pick a cost-effective dominating set of the question graph. In summary, we have a tendency to create the subsequent contributions: 1) we have a tendency to style a unique strategy to with efficiency method SMS2 queries. associate inverted pattern lattice primarily based classification and a structural signature-based neck of the woods sensitive hashing ar 1st made offline. throughout the web part, a group of pruning techniques[3] expedited by the offline knowledge structures ar introduced and integrated along to greatly scale back thesearch house of SMS2 queries. 2) we have a tendency to propose set similarity 
pruning techniques that utilize a unique inverted pattern lattice over the component sets of knowledge vertices to judge dynamic weighted set similarity. It introduces associate bound on the dynamic weighted similarity live to use the anti-monotone principle to realize high pruning power 3) we have a tendency to propose structure-based pruning techniques that explore a unique structural- signature-based system, wherever the signature is meant to capture the set and neighborhood info. associate mixture dominance[4] principle is devised to guide the pruning 4) rather than directly questioning and corroboratory the candidates of all the vertices within the query graph, we have a tendency to style associate economical formula to perform subgraph matching supported the dominating set of question graph. once filling up the remaining (non-dominating) vertices of the graph, a distance preservation principle is devised to prune candidate vertices that don't preserve the space to dominating vertices. 5) Last however not least, we have a tendency to demonstrate through in depth experiments that our approach will effectively and with efficiency answer the SMS2 queries in an exceedingly massive graph information.

\section{IMPLEMENTATION}

\section{MODULE DESCRIPTION:}

\section{Number of Modules:}

After careful analysis the system has been known to own the subsequent modules

1. Framework

2. Set similarity pruning

3. Pruning Techniques

4. $\quad$ Structure-based pruning

5. Dominating-set-based subgraph matching

\section{Framework}

In this segment, we have a tendency to gift a filter-and-refine framework for our projected approaches, which incorporates offline process and on-line process. Offline processing: we have a tendency to construct a unique inverted pattern lattice to facilitate economical pruning supported the set similarity. Since the dynamic weight of every component makes existing indices inefficient for responsive SMS2 queries, we want to style a unique index for SMS2 
question. The lattice along side the inverted lists is termed inverted[5] pattern lattice, which may greatly scale back the value of dynamic weighted set similarity search. To support structure-based pruning, we have a tendency to write in code every question vertex and information vertex into a question signature and a knowledge\} signature severally by considering each the set and topology information, and hash all the info signatures into signature buckets. Online processing: we have a tendency to propose finding a cost-effective dominating set (defined in Section 6) of the question graph alphabetic character, and solely search candidates for vertices within the dominating set. Note that, completely different|completely different\} dominating sets can cause different question performances. Thus, we have a tendency to propose a dominating set choice algorithmic program to pick out a cost-effective dominating set $\mathrm{DS}(\mathrm{Q})$ of question graph alphabetic character. The dominating-set-based subgraph matching is motivated by 2 observations: (1) finding candidates in SMS2 queries is far costlier than that in typical subgraph search, as a result of set similarity calculation is a lot of pricey than vertex label matching. As a result, the filtering value will be reduced by looking dominating vertices of $\mathrm{V}(\mathrm{Q})$ instead of all question vertices. (2) Some question vertices might have an outsized quantity of candidate vertices, that results in several excess intermediate results throughout subgraph matching. Therefore, the ubgraph matching value may also be reduced by decreasing the scale of intermediate results. for every vertex $u$ two $\mathrm{DS}(\mathrm{Q})$, we have a tendency to propose a twophase pruning strategy, together with set similarity pruning and structure-based pruning. The set,similarity pruning includes anti-monotone pruning, vertical pruning, and horizontal pruning, that ar supported our projected inverted pattern lattice. supported the signature buckets, we have a tendency to conjointly propose the structure-based pruning technique by utilizing novel vertex signatures. once the pruning, we have a tendency to propose associate economical DS-Match subgraph matching algorithmic program to get subgraph matches of alphabetic character supported candidates of dominating vertices in DS(Q) (Section 6). DS-match utilizes topological relations between dominating vertices and non-dominating vertices to scale back the size of intermediate results throughout subgraph matching, and so reduces the matching value. Set similarity pruning For a vertex $\mathrm{u}$ during a dominating set of question graph alphabetic character, we want to seek out its candidate vertices in graph G. allow us to recall the definition of SMS2 question. If a vertex $\mathrm{v}$ in graph $\mathrm{G}$ match with $\mathrm{u}, \operatorname{sim}(\mathrm{S}(\mathrm{u}) ; \mathrm{S}(\mathrm{v}))$ \&gt; nine holds. This section concentrates on finding candidate vertices $v$ of $u$ specified $\operatorname{sim}(\mathrm{S}(\mathrm{u}) ; \mathrm{S}(\mathrm{v}))$ \&gt; . Existing indices wishing on component canonicalization don't seem to be appropriate for SMS2 queries thanks to dynamic weights of components. even so, we have a tendency to note that the inclusion 
relation between 2 sets doesn't modification notwithstanding component weights vary dynamically. for 2 component sets $S(v)$ and $S(v 0)$ of vertex $v$ and $v 0$ severally, if $S(v)$ nine $\mathrm{S}(\mathrm{v} 0)$, the connection of $\mathrm{S}(\mathrm{v})$ being a set of $\mathrm{S}(\mathrm{v} 0)$ is termed inclusion relation.

\section{Pruning Techniques}

-Anti-monotone Pruning

Considering the anti-monotone property of AS edge and also the characteristics of inverted lattice pattern, we've the subsequent theorem. Theorem 1: Given a question vertex $\mathrm{u}$, for every accessed frequent pattern $\mathrm{P}$ within the inverted pattern lattice, if $\mathrm{UB}(\mathrm{S}(\mathrm{u})$; $\mathrm{P})$ \&lt; nine , all vertices within the inverted list $\mathrm{L}(\mathrm{P})$ and $\mathrm{L}(\mathrm{P} 0)$ are often safely cropped, wherever $\mathrm{P} 0$ may be a descendant node of $\mathrm{P}$ within the lattice.

Proof: for every part set $S(v)$ within the inverted list of $P$, since $P$ nine $S(v), U B(S(u)$; $S(v))$ \&lt; nine in line with the anti-monotone property of AS edge. Similarly, for any descendant node $\mathrm{P} 0$ of $\mathrm{P}$, since $\mathrm{P} 0$ nine $\mathrm{P}, \mathrm{UB}(\mathrm{S}(\mathrm{u})$; $\mathrm{P} 0)$ are additionally[6] but nine . the theory are often well-tried. supported the theory on top of, we will with efficiency prune frequent patterns within the inverted pattern lattices notwithstanding dynamic weights of components.

\section{-Vertical Pruning}

Vertical pruning relies on the prefix filtering principle [30]: if 2 canonicalized sets square measure similar, the refixes of those 2 sets ought to overlap with one another, as otherwise these 2 sets won't have enough common components. we have a tendency to canonicalize all the weather in question set $\mathrm{S}(\mathrm{u})$ during a descendant order of their weights throughout on-line process. the primary $\mathrm{p}$ components within the canonicalized set $S(\mathrm{u})$ is denoted because the $\mathrm{p}$ prefix of $S(\mathrm{u})$. we discover the most prefix length $\mathrm{p}$ such if $\mathrm{S}(\mathrm{u})$ and $\mathrm{S}(\mathrm{v})$ haven't any overlap in $\mathrm{p}$ - refix, $\mathrm{S}(\mathrm{v})$ are often safely cropped, as a result of they are doing not have enough overlap to satisfy the similarity threshold [30]. to seek out p-prefix of $S(u)$, when we have a tendency to take away the part with the biggest weight from $S(\mathrm{u})$, we have a tendency to check whether or not the remaining set $\mathrm{SO}(\mathrm{u})$ meets the similarity threshold with $\mathrm{S}(\mathrm{u})$. $\mathrm{P}$ we have a tendency to denote L1-norm of $\mathrm{S}(\mathrm{u})$ as $\mathrm{kS}(\mathrm{u}) \mathrm{k} 1=\mathrm{a} 2 \mathrm{~S}(\mathrm{u}) \mathrm{W}(\mathrm{a})$. If $\mathrm{kSO}(\mathrm{u}) \mathrm{k} 1$ \&lt; nine nine $\mathrm{kS}(\mathrm{u}) \mathrm{k} 1$, the removal stops. the worth of $\mathrm{p}$ is adequate $\mathrm{jS} 0(\mathrm{u}) \mathrm{j} \square 1$, wherever $\mathrm{jSO}(\mathrm{u}) \mathrm{j}$ is that the range of components in $\mathrm{S} 0(\mathrm{u})$. For any set $\mathrm{S}(\mathrm{v})$ that doesn't contain the weather in $\mathrm{S}(\mathrm{u})^{\prime} \mathrm{s}$ p-prefix, we've $\operatorname{sim}(\mathrm{S}(\mathrm{u})$; $\mathrm{S}(\mathrm{v}))$ nine $\mathrm{kSO}(\mathrm{u}) \mathrm{k} 1 \mathrm{kS}(\mathrm{u}) \mathrm{k} 1$ \&lt; nine, thus $\mathrm{S}(\mathrm{u})$ and $\mathrm{S}(\mathrm{v})$ won't meet the set similarity threshold. 
Theorem 2: Given a question set $\mathrm{S}(\mathrm{u})$ and a frequent pattern $\mathrm{P}$ within the lattice, if $\mathrm{P}$ isn't a 1- frequent pattern (or its descendant) in $S(\mathrm{u})^{\prime} \mathrm{s}$ p-prefix, all vertices within the inverted list L(P) are often safely cropped.

Proof: for every vertex $\mathrm{v}$ in $\mathrm{L}(\mathrm{P}), \mathrm{P}_{-} \mathrm{S}(\mathrm{v})$. in line with prefix filtering principle and Theorem one, all vertices in $\mathrm{L}(\mathrm{P})$ are often cropped. when we discover the p-prefix of $\mathrm{S}(\mathrm{u})$, we have a tendency to solely have to be compelled to access all the descendent nodes of the corresponding 1- frequent patterns in p-prefix during a vertical manner.

\section{-Horizontal Pruning}

Intuitively, a question part set $S(\mathrm{u})$ can't be the same as a frequent pattern of an outsized size set or a frequent pattern of a really little size. the dimensions of a frequent pattern $\mathrm{P}$ (denoted by $\mathrm{jP}$ ) is that the range of components in $\mathrm{P}$. within the inverted pattern lattice, every frequent pattern $\mathrm{P}$ may be a set of knowledge vertices (i.e., part sets) in $\mathrm{P}^{\prime} \mathrm{s}$ inverted list. Suppose we will notice the length edge for $S(u)$ (denoted by $L U(u)$ ). If the dimensions of $P$ is larger than $\mathrm{LU}(\mathrm{u})$, (i.e. the sizes of all information vertices in $\mathrm{P}^{\prime} \mathrm{s}$ inverted list square measure larger than $\mathrm{LU}(\mathrm{u}))$ then $\mathrm{P}$ and its inverted list are often cropped. as a result of dynamic part weights, we'd like to seek out $S(\mathrm{u})^{\prime} \mathrm{s}$ length interval on the fly. we discover $\mathrm{LU}(\mathrm{u})$ by adding components in $(\mathrm{U} \square \mathrm{S}(\mathrm{u}))$ to $\mathrm{S}(\mathrm{u})$ in Associate in Nursing increasing order of their weights. when a part is additional, a brand new set $\mathrm{SO}(\mathrm{u})$ is created. we have a tendency to calculate the similarity price between $\mathrm{S}(\mathrm{u})$ and $\mathrm{SO}(\mathrm{u})$. If $\operatorname{sim}(\mathrm{S}(\mathrm{u})$; $\mathrm{SO}(\mathrm{u})$ ) nine nine holds, we have a tendency to still add components to $\mathrm{SO}(\mathrm{u})$. Otherwise, the edge $\mathrm{LU}(\mathrm{u})$ equals to $\mathrm{jSO}(\mathrm{u}) \mathrm{j} \square$ one. Note that, frequent patterns at constant level of the inverted pattern lattice have constant size, and also the size of frequent patterns at Level $k$ equals to $k$. Thus, when getting the length edge $\mathrm{LU}(\mathrm{u})$ of $\mathrm{S}(\mathrm{u})$, we will confirm that the horizontal edge equals to $\mathrm{LU}(\mathrm{u})$. All frequent patterns beneath Level $\mathrm{LU}(\mathrm{u})$ are cropped.

-Putting All Pruning Techniques along

In this segment, we have a tendency to apply all the set similarity pruning techniques and acquire candidates for a question vertex. for every question vertex $u$, we have a tendency to 1st use vertical pruning and horizontal pruning to filter false positive patterns and collectively confirm the nodes (i.e., frequent patterns) that ought to be accessed within the inverted pattern lattice. Then, we have a tendency to traverse them during a breadth-first manner. for every accessed node $\mathrm{P}$ within the lattice, we have a tendency to check whether or not $\operatorname{UB}(\mathrm{S}(\mathrm{u})$; $\mathrm{P})$ is a smaller amount than nine. If yes, $\mathrm{P}$ and its descendant nodes square measure cropped 
safely. The gray nodes are often cropped on balance pruning techniques on top of are applied. Assume that $\mathrm{P} 1, \mathrm{P} 2, \ldots$, and $\mathrm{Pk}$ square measure the remaining nodes (i.e., white nodes) that can't be cropped, the candidate set of $u$ may be a set of Sunflower State $i=1 \mathrm{~L}(\mathrm{Pi})$.

\section{Structure-based pruning}

A matching subgraph shouldn't solely have its vertices (element sets) like that in question graph letter of the alphabet, however conjointly preserve constant structure as letter of the alphabet. Thus, during this section, we have a tendency to style light-weight signatures for each question vertices and information vertices to more filter the candidates when set similarity pruning by considering the structural info.

\section{Structural Signatures}

We outline 2 distinct forms of structural signature, particularly question signature $\operatorname{Sig}(\mathrm{u})$ and information signature $\operatorname{Sig}(\mathrm{v})$ for every question vertex $\mathrm{u}$ and information vertex $\mathrm{v}$, severally. To write structural info, $\operatorname{Sig}(\mathrm{u})=\operatorname{Sig}(\mathrm{v})$ ought to contain the component info of each $\mathrm{u}=\mathrm{v}$ and its close vertices. Since the question graph is typically tiny, we have a tendency to generate correct question signatures by encryption every neighbor vertex individually. On the contrary, the information graph is way larger than the question graph, therefore the aggregation of neighbor vertices will save lots of area. The pruning value are often conjointly reduced owing to restricted variety of information signatures.

\section{Signature-based LSH}

To alter economical pruning supported structural info, we have a tendency to use a collection of neck of the woods Sensitive Hashing (LSH) [31] hash functions to hash every information signature $\operatorname{Sig}(\mathrm{v})$ into a signature bucket, that is outlined as follows.

\section{Structural Pruning}

Based on the SMS2 question definition (Definition 1), a knowledge vertex $\mathrm{v}$ are often cropped if the similarity between BV (u) and BV (v) is smaller than nine, or there doesn't exist BV (vj) that satisfies the similarity constraint with BV (ui), wherever vj $(j=1 ;:: ; n$ ) and ui $(\mathrm{i}=1 ;: . ; ; \mathrm{m})$ ar one-hop neighbors of $\mathrm{v}$ and $\mathrm{u}$, severally. we have a tendency to outline the 
similarity between BV (u) and BV (v) as follows, that is analogous to the weighted Jaccard similarity.

\section{Dominating-set-based subgraph matching}

In this section, we have a tendency to propose associate economical dominating-set(DS)based mostly subgraph matching formula (denoted by DS-Match) expedited by a dominating set choice technique.

\section{DS-Match formula}

DS-Match formula initial finds matches of a dominating question graph QD (defined in Definition 14) shaped by the vertices in dominating set $D S(Q)$, then verifies whether or not every match of letter of the alphabetD are often extended as a match of Q. DSMatch is actuated by 2

observations: initial, compared with typical subgraph matching over vertex-labeled graph, the overhead of finding candidates in SMS2 queries is comparatively higher, because the computation value of set similarity is way above that of label matching. we will save filtering value by solely finding candidate vertices for dominating vertices instead of all vertices in letter of the alphabet. Second, we will speed up subgraph matching by solely finding matches of dominating question vertices. The candidates of remaining (non-dominating) question vertices are often stuffed up by the structural constraints between dominating vertices and nondominating vertices. during this manner, the dimensions of intermediate results throughout subgraph matching are often greatly reduced.CONCLUSIONS

In this paper, we tend to study the matter of subgraph matching with set similarity, that exists in a very big selection of applications. To tackle this downside, we tend to propose economical pruning techniques by considering each vertex set similarity and graph topology. a completely unique inverted pattern lattice and structural signature buckets area unit designed to facilitate the web pruning. Finally, we tend to propose AN economical dominating-setbased subgraph match formula to seek out subgraph matches. intensive experiments are conducted to demonstrate the potency and effectiveness of our approaches compared to progressive subgraph matching ways. 


\section{REFERENCES}

[1] L. Zou, L. Chen, and M. T. Ozsu, "Distance-join: Pattern match query in a large graph database," PVLDB, vol. 2, no. 1, 2009.

[2] J. Cheng, J. X. Yu, B. Ding, P. S. Yu, and H. Wang, "Fast graph pattern matching," in Data Engineering, 2008. ICDE 2008. IEEE 24th International Conference on. IEEE, 2008, pp. 913 - 922.

[3] Y. Tian and J. M. Patel, "Tale: A tool for approximate large graph matching," in ICDE, 2008.

[4] S. Brucknerl, F. Huffner, R. M. Karp, R. Shamir, and R. Sharan, "Torque: topology-free querying of protein interaction networks," Nucleic Acids Research, vol. 37, no. suppl 2, pp. W106-W108, 2009.

[5] Y. Tian, R. C. McEachin, C. Santos, D. J. States, and J. M. Patel, "Saga: a subgraph matching tool for biological graphs," Bioinformatics, vol. 23, no. 2, pp. 232-239, 2007.

[6] P. Zhao and J. Han, "On graph query optimization in large networks," PVLDB, vol. 3, no. 1-2, 2010. 